\title{
Adverse events in women and children who have received intrapartum antibiotic prophylaxis treatment: a systematic review
}

\author{
Farah Seedat ${ }^{1}$, Chris Stinton ${ }^{1}$, Jacoby Patterson ${ }^{1}$, Julia Geppert ${ }^{1}$, Bee Tan ${ }^{1,2}$, Esther R. Robinson ${ }^{3}$,
}

Noel Denis McCarthy ${ }^{1}$, Olalekan A. Uthman ${ }^{1}$, Karoline Freeman ${ }^{1}$, Samantha Ann Johnson' ${ }^{1}$, Hannah Fraser ${ }^{1}$, Colin Stewart Brown ${ }^{4}$, Aileen Clarke ${ }^{1}$ and Sian Taylor-Phillips ${ }^{1 *}$ (1)

\begin{abstract}
Background: Adverse events from intrapartum antibiotic prophylaxis (IAP) are poorly documented yet essential to inform clinical practice for neonatal group B Streptococcus (GBS) disease prevention. In this systematic review, we appraised and synthesised the evidence on the adverse events of IAP in the mother and/or her child.

Methods: We searched MEDLINE, MEDLINE In-Process \& Other Non-Indexed Citations, EMBASE, Cochrane, and Science Citation Index from date of inception until October 16th 2016. Reference lists of included studies and relevant systematic reviews were hand-searched. We included primary studies in English that reported any adverse events from intrapartum antibiotics for any prophylactic purpose compared to controls. The search was not restricted to prophylaxis for GBS but excluded women with symptoms of infection or undergoing caesarean section. Two reviewers assessed the methodological quality of studies, using the Cochrane Risk of Bias tool, and the Risk of Bias Assessment Tool for Nonrandomised Studies. Results were synthesised narratively and displayed in text and tables.

Results: From 2364 unique records, 30 studies were included. Despite a wide range of adverse events reported in 17 observational studies and 13 randomised controlled trials (RCTs), the evidence was inconsistent and at high risk of bias. Only one RCT investigated the long-term effects of IAP reporting potentially serious outcomes such as cerebral palsy; however, it had limited applicability and unclear biological plausibility. Seven observational studies showed that IAP for maternal GBS colonisation alters the infant microbiome. However, study populations were not followed through to clinical outcomes, therefore clinical significance is unknown. There was also observational evidence for increased antimicrobial resistance, however studies were at high or unclear risk of bias.
\end{abstract}

Conclusions: The evidence base to determine the frequency of adverse events from intrapartum antibiotic prophylaxis for neonatal GBS disease prevention is limited. As RCTs may not be possible, large, better quality, and longitudinal observational studies across countries with widespread IAP could fill this gap.

Trial registration: CRD42016037195.

Keywords: Streptococcus agalactiae, Group B Streptococcus, Intrapartum antibiotic prophylaxis, Adverse events, Harms, Systematic review

\footnotetext{
* Correspondence: S.Taylor-Phillips@warwick.ac.uk

'Division of Health Sciences, University of Warwick Medical School, Gibbet

Hill Campus, Coventry CV4 7AL, UK

Full list of author information is available at the end of the article
} International License (http://creativecommons.org/licenses/by/4.0/), which permits unrestricted use, distribution, and reproduction in any medium, provided you give appropriate credit to the original author(s) and the source, provide a link to the Creative Commons license, and indicate if changes were made. The Creative Commons Public Domain Dedication waiver (http://creativecommons.org/publicdomain/zero/1.0/) applies to the data made available in this article, unless otherwise stated. 


\section{Background}

Group B Streptococcus (GBS), or Streptococcus agalactiae, a gram-positive bacterium, is the leading cause of mortality and morbidity from neonatal sepsis [1]. GBS colonises the gastrointestinal and/or genitourinary tract in 10 to $30 \%$ of pregnant women [2-4], with a recent global rate of $17.9 \%$ [5]. If a pregnant woman is vaginally colonised with GBS when she is in labour, there is a $36 \%$ chance that GBS will be transmitted to her neonate [6]. Most GBS colonised neonates will be asymptomatic, however less than $1 \%$ may suffer from invasive early-onset GBS disease (less than seven days, EOGBS) [7]. Globally, cultureconfirmed EOGBS has an estimated incidence of 0.43 per 1000 live births and a case fatality rate of $12.1 \%$, which may be an underestimate [8].

To prevent EOGBS, the currently available prevention is intrapartum antibiotic prophylaxis (IAP), administered to mothers identified at risk of vertically transmitting GBS bacteria $[9,10]$. The current recommendation for IAP in Western Europe, North America, and Australasia is intravenous penicillin (or ampicillin) given as soon as possible after the onset of labour and then every four hours until delivery, with intravenous cefazolin in the US, or clindamycin in the UK, for mothers allergic to penicillin [9-11]. Pregnant women are selected to be offered IAP using different policies. In some countries, women are offered IAP if they present with known risk factors for GBS, such as intrapartum fever or GBS bacteriuria, and in many other countries, women are actively screened for GBS colonisation at 35-37 weeks of pregnancy and treated in labour if they are positive [12]. Screening for GBS maternal colonisation is controversial as up to $30 \%$ of women with positive results at 35-37 weeks revert to negative by labour [13], and the large majority of women who are colonised with GBS during labour have healthy neonates who will not suffer from EOGBS.

A number of potential harms have been suggested as a result of the widespread use of IAP associated with neonatal GBS prevention $[14,15,10]$. IAP has been associated with antimicrobial resistance [16-18], neonatal infections caused by gram-negative bacteria $[19,15,18]$, Clostridium difficile infection in mothers [20], maternal anaphylaxis, which although very rare, can be fatal for mother and baby [9], neonatal microbiota changes that could lead to short and long-term health problems [21-23], anxiety for the mother, family, and medical staff, and the medicalisation of labour $[15,10]$.

The potential harms from IAP are poorly documented and understood, and there has been no systematic review of the evidence. This information is essential to assess whether the benefits of IAP treatment for neonatal GBS disease prevention outweigh the harms. Therefore, we conducted a systematic review to identify, appraise, and synthesise the evidence on the adverse events experienced by the mother and/or her child after receiving IAP treatment. This review was conducted as part of a national review on whether the UK should introduce a GBS screening programme, a crucial part of these screening reviews is to understand the harms of treatment.

\section{Methods}

This systematic review is reported according to PRISMA guidelines [24]. As this was a secondary analysis of existing data, ethical consent was not required.

\section{Search strategy}

Searches were conducted in MEDLINE, MEDLINE InProcess \& Other Non-Indexed Citations, EMBASE, Cochrane Library: Cochrane Database of Systematic Reviews, CENTRAL, DARE and HTA databases, and Science Citation Index Expanded from date of inception until October 16th 2016. The search strategy combined both text words and MeSH terms for antibiotic prophylaxis, labour, and adverse events, and was limited to English and humans (see Additional file 1 for complete search strategy). We used recommended search filters for adverse events [25, 26], and systematically included terms for known IAP adverse events from previous studies $[14,15,27,10]$ and expert opinion. We also hand-searched reference lists of included studies and relevant systematic reviews, and experts cross-checked included studies.

\section{Eligibility criteria and study selection}

Two reviewers independently screened the titles, abstracts, and full texts of all identified records. Disagreements were resolved by discussion, with involvement of a third reviewer if necessary. We included any full text randomised controlled trials (RCTs), cohort studies, or case-control studies in English, reporting any adverse events experienced by mothers and/or their children after being exposed to antibiotics during labour for any prophylactic purpose, compared to an unexposed control group. As the evidence base on IAP for neonatal GBS disease prevention is limited, we included studies on IAP for any prophylactic indication. IAP studies for caesarean sections or symptomatic mothers, those in which women were given antibiotics before labour, or neonates given antibiotics after birth were excluded. Studies were included if $90 \%$ or more of the study population met the inclusion criteria, or if results for those who met the inclusion criteria were reported separately. We excluded case series, case reports, abstracts, editorials, letters, books, consensus statements, opinions, and reviews. 


\section{Quality appraisal}

Two reviewers independently appraised the risk of bias for each included study using the Cochrane Risk of Bias (RoB) tool [28], and the Risk of Bias Assessment Tool for Nonrandomised Studies (RoBANS) [29]. Selection, performance, detection, attrition, and reporting biases were assessed and classified as low, high, and unclear risk of bias.

\section{Data extraction and synthesis}

Meta-analyses could not be performed due to the heterogeneity across the adverse outcomes assessed. Narrative syntheses were conducted, and the results of individual studies displayed in text and tables. Missing statistical parameters of importance were calculated if data permitted. Odds ratios (ORs) were calculated for case-control studies and risk ratios (RRs) and risk differences (RDs) were calculated for all other study designs using Stata version 13 (Stata Corp, College Station, Texas).

\section{Results}

\section{Characterisation of included studies}

Our search identified 2364 unique references. After sifting titles and abstracts, 262 full texts were screened, of which 30 studies met the inclusion criteria and were included in the synthesis (see Fig. 1 for study flow and Additional file 1 for full text studies excluded with reason) [30-59]. Fourteen were cohort studies $[30,32,33,46,35-37,39,42,44,45,56,31,57]$, three casecontrol [34, 38, 54], 12 RCTs [40, 47-53, 55, 43, 41, 58], and one a sub-study [59] of an included RCT [58]. Nine studies investigated IAP for GBS prevention [30, 34, 46, 35, $36,39,54,31,57]$, two for GBS prevention and other indications [37, 42], three for post-partum infection prevention $[40,49,50]$, eight for preterm labour $[41,43,47,48,51-53$, 55], two for neonatal sepsis prevention [58, 59], and six did not state the indication (see Additional file 1: Table S1 for study characteristics) $[32,33,38,45,44,56]$. Some IAP effectiveness trials reported outcomes, such as neonatal and maternal infection, that could plausibly increase from IAP due to changes in the organisms causing infections and/or antibiotic susceptibility [18, 44], but could also decrease if the IAP is successful $[40,43,47-53,55,58]$. To prevent bias in reporting, we reported these outcomes irrespective of whether they were identified as benefits or harms (see bottom of Additional file 1: Table S1).

\section{Methodological quality}

None of the RCTs were judged as low risk of bias across all domains as assessed by the Cochrane Risk of Bias tool (see Fig. 2) [28]. The greatest risk of bias was in selective outcome reporting, where eight RCTs were at high risk partly or solely because the definition and measurement of side effects was not pre-specified in the methods but only reported in the results $[47-50,53,55,40,59]$. More than half of the RCTs were rated as having unclear risk of bias for incomplete outcome data as there was substantial missing data, for example, on adverse events in the control group [40, 49-51, 53, 58, 55]. We noted a number of other sources of bias across RCTs, including relatively small sample sizes $[41,52,59]$, data not presented $[41,49,50]$, a lack of information on treatment regimens [48] and details of intention to treat analysis [51, 55], inaccuracies in the numbers provided for participant flow [53], and parent-reported outcomes rather than objective assessment [41].

There were no observational studies judged as low risk of bias across all domains on the RoBANS tool (see Fig. 3) [29]. The confounding variables domain had the highest concern, as four studies were rated as high risk [30, 37, 44, 46], none as low risk, and 13 as unclear risk of bias [32-36, $38,39,42,45,54,56,31,57]$, as some variables were accounted for in the study design or at least reported, while others, such as maternal risk factors, prenatal antibiotics, and caesarean sections, were not. Likewise, selection of participants was also unclear across nine studies [32, 33, 39, $44,45,54,56,31,57]$, as there was no mention of how participants were selected and/or some important baseline characteristics were not reported.

\section{Adverse events associated with IAP}

A range of child and maternal adverse events were investigated for association with IAP including maternal thrush, childhood atopic dermatitis, neonatal infections and respiratory distress, necrotising enterocolitis, and Clostridium difficile bowel problems (see Additional file 1: Table S1 for summary of results). Below we present the findings on three key results - gut microbiota, antibiotic resistance, and long-term adverse events.

\section{Gut microbiota}

Seven cohort studies consistently showed that IAP alters the infant microbiome [30, 32, 33, 36, 39, 31, 57]. At day $2,3,6-7,10,30$, and 90 , there were differences in the relative composition and the colony forming units per gram $(\log \mathrm{CFU} / \mathrm{g})$ of organisms in the gut of infants whose mothers were and were not treated with IAP (see Tables 1 and 2 for results) [32, 33, 39, 31, 57, 36]. Two studies also reported on sample richness and biodiversity, finding that at day 6-7 and day 30 , infants whose mothers were treated with IAP had a less diverse microbial profile compared to controls [31, 57]. At day 6-7, there was also a clear segregation between the microbiota profiles of IAP compared to control infants when they were plotted on principal coordinate analysis plots, which disappeared by day 30 [31, 57]. Similar to gut microbiota, Keski-Nisula et al. (2013), [42] found a decreased transmission of vaginal Lactobacillus-dominant 
Records identified through database searching

( $n=3625$ )
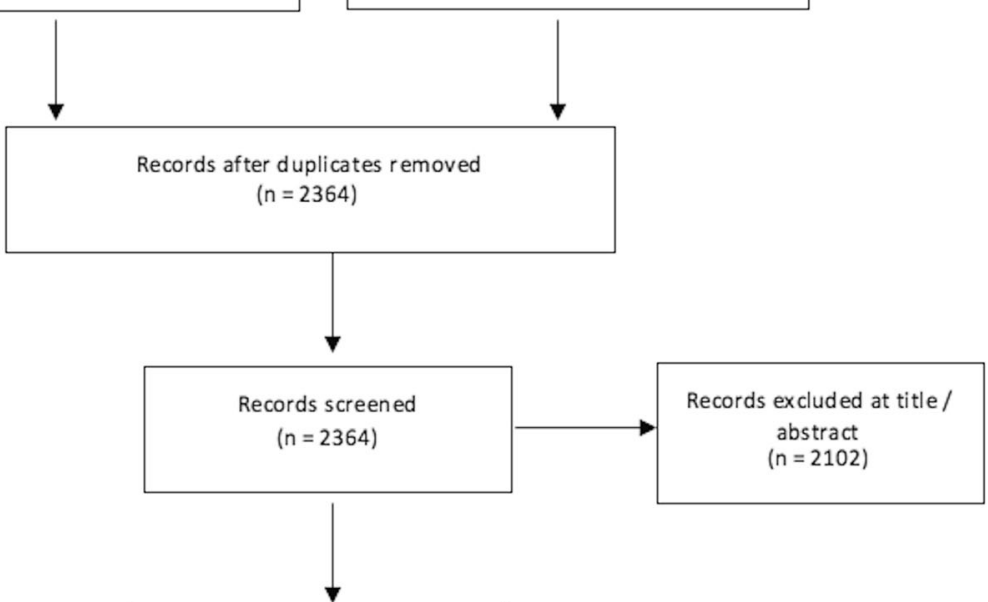

Full-text articles assessed for

eligibility

( $n=262$ )

Additional records identified through

other sources

$(n=13)$

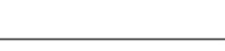

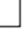
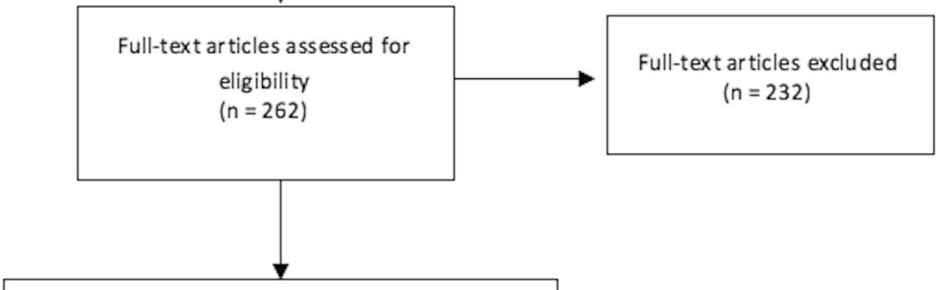

Studies included in synthesis $(n=30)$

Fig. 1 Flow diagram of study selection 
Risk of bias

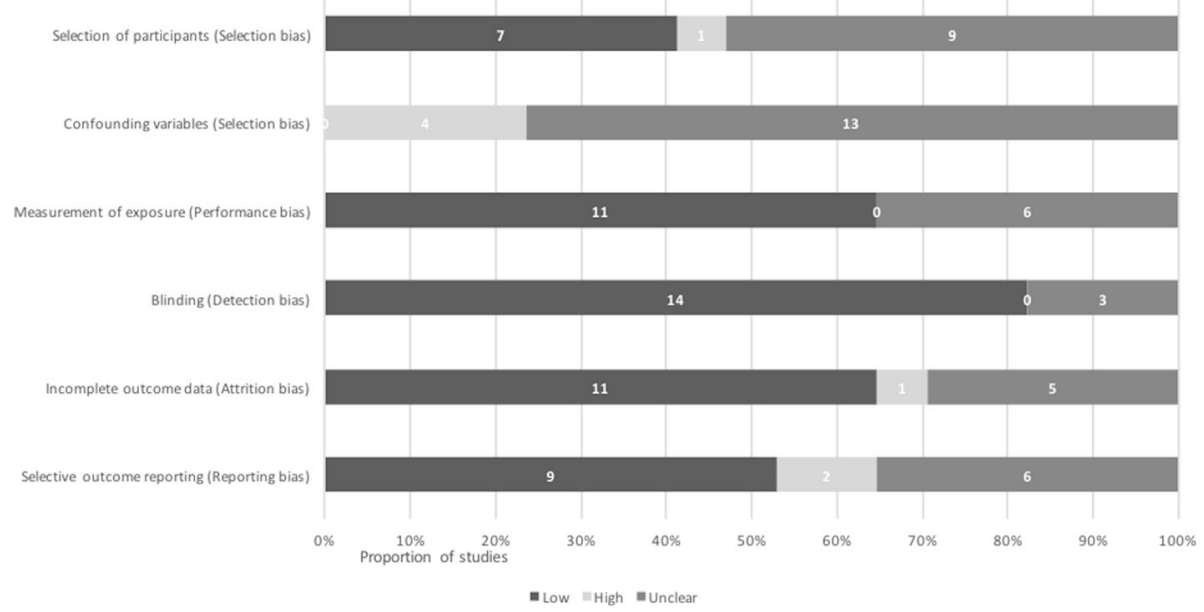

Fig. 3 Risk of bias in non-randomised studies according to RoBANS [29]

mixed flora on oral surfaces in neonates whose mothers were treated with IAP compared to those who were not ( 1 versus $13, \mathrm{OR}=0.0895 \% \mathrm{CI} 0.007-0.80$ ). While there was consistent evidence on gut microbiota alterations, it is unclear if any of the alterations are related to clinical adverse events or not.

\section{Antimicrobial resistance}

Six studies reported antimicrobial resistance. Of the RCTs, Gordon et al. (1995) reported zero cases of multi-resistant bacterial infections in the intervention group of 58 infants whose mothers were treated with IAP for preterm labour [48]. Roca et al. (2016) investigated GBS, Staphylococcus aureus (S. aureus), and Streptococcus pneumoniae ( $S$. pneumoniae) resistant to azithromycin in 829 mothers and 843 infants treated with azithromycin for neonatal sepsis prevention [58]. S. aureus resistant to azithromycin were found at day 3 in maternal breast milk, at day 6 in newborn nasopharynx and maternal breast milk, at day 8 in vaginal swabs, and at day 14 and day 28 in newborn and maternal nasopharynx and maternal breast milk. $S$. pneumoniae resistant to azithromycin were also identified in the maternal nasopharynx, which occurred at day 28 only (see Additional file 1: Table S1).

Of the four observational studies, Glasgow et al. (2005) found that in 62 infants whose mothers were treated with various IAP drugs (indication not stated), 24 (39\%) had ampicillin-resistant organisms, compared to 13/120 (11\%) infants whose mothers were not treated $(\mathrm{OR}=5.795 \% \mathrm{CI}$ 2.3-14.3) [38]. The authors also reported a significant difference when analysing ampicillin-resistant bacteria causing urinary tract infections separately $(\mathrm{OR}=4.395 \% \mathrm{CI}$ 1.6-11.7). Similarly, Stoll et al. (2002) found that mothers of infants with ampicillin-resistant strains of Escherichia coli $(E$. coli) were more likely to have received intrapartum ampicillin than those with ampicillin-sensitive strains (26 of 28 [93\%] versus 1 of 5 [20\%] $p=0.01$ ) [44]. It was unclear whether the infants in these two studies were treated with antibiotics before they were tested for antimicrobial resistance. Ashkenazi-Hoffnung et al. (2011) did not find any differences between 17 infants born to mothers treated with IAP for GBS prevention and 178 infants who were not, in first generation cephalosporin resistance in $E$. coli $(60 \%$ versus $22.7 \% p=0.21)$ or any bacteria causing late-onset serious bacterial infections (57\% versus $26 \%$ $p=0.19)$, or ampicillin resistance in $E$. coli $(100 \%$ versus $54.5 \% p=0.14$ ) or any bacteria causing late-onset serious bacterial infections $(85 \%$ versus $63 \% p=0.19)$ [34]. The authors did find higher development of first generation cephalosporin-resistant urinary tract infections (75\% versus $23.5 \% p=0.04)$. Lastly, Jaureguy et al. (2004) did not find a difference in the number of infants colonised with amoxicillin-resistant Enterobacteriaceae $(10 / 25$ [40\%] versus $12 / 25$ [48\%], calculated $\mathrm{RR}=0.8395 \% \mathrm{CI}$ 0.44-1.56) and amoxicillinresistant E. coli (6/25 [24\%] versus 11/25 [44\%] calculated $\mathrm{RR}=0.5595 \% \mathrm{CI} 0.24-1.25)$ in the gut of infants whose mothers were or were not treated with IAP [39].

\section{Long-term adverse events}

Kenyon et al. (2008) was the only RCT that reported on the long-term adverse events of IAP [41]. They found that IAP may be associated with severe consequences of functional impairment and cerebral palsy, as well as bowel problems in a factorial randomised trial comparing children aged seven whose mothers had received any erythromycin (erythromycin alone or combined with amoxicillin-clavulanate) compared to no erythromycin, and any amoxicillin-clavulanate (alone or with erythromycin) 
Table 1 Qualitative gut microbiota composition of IAP-treated and untreated infants

\begin{tabular}{|c|c|c|c|c|c|c|c|c|}
\hline \multirow[t]{2}{*}{ Organism } & \multirow[t]{2}{*}{ Study } & \multirow{2}{*}{$\begin{array}{l}\text { Number of } \\
\text { infants }(n) \text { in } \\
\text { each group }\end{array}$} & \multicolumn{6}{|c|}{ Relative abundance in microbiota composition (\%) or number of infants $(n)$ colonised } \\
\hline & & & Day 1 & Day 2 & Day 3 & Day 6/7 & Day 10 & Day 30 \\
\hline \multicolumn{9}{|l|}{ Phyla } \\
\hline All & $\begin{array}{l}\text { Arboleya } 2016 \\
\text { [33] }\end{array}$ & $\begin{array}{l}\text { IAP } n=14 \\
\text { Control } n=13\end{array}$ & $\begin{array}{l}\text { No } \\
\text { differences }\end{array}$ & & & & & \\
\hline \multirow[t]{4}{*}{ Actinobacteria } & $\begin{array}{l}\text { Aloisio } 2016 \\
{[31]^{a}}\end{array}$ & $\begin{array}{l}\text { IAP } n=10 \\
\text { Control } \\
n=10\end{array}$ & & & & $\begin{array}{l}\text { IAP: } 0.4 \% \\
\text { Control: } 3.8 \% \\
p<0.05\end{array}$ & & \\
\hline & $\begin{array}{l}\text { Arboleya } 2016 \\
\text { [33] }\end{array}$ & $\begin{array}{l}\text { IAP } n=14 \\
\text { Control } \\
n=13\end{array}$ & & & & & & $\begin{array}{l}\text { Lower } \% \text { in IAP, } \\
p<0.05\end{array}$ \\
\hline & $\begin{array}{l}\text { Mazzola } 2016 \\
{[57]^{a}}\end{array}$ & $\begin{array}{l}\text { Breast-fed IAP } \\
n=7 \\
\text { Breast-fed } \\
\text { Control } n=7\end{array}$ & & & & $\begin{array}{l}\text { IAP: } 0 \% \\
\text { Control: } 17 \% \\
p<0.001\end{array}$ & & \\
\hline & & $\begin{array}{l}\text { Mixed-fed IAP } \\
n=6 \\
\text { Mixed-fed } \\
\text { Control } \\
n=6\end{array}$ & & & & $\begin{array}{l}\text { IAP: } 1 \% \\
\text { Control: } 8 \% \\
\text { RR } 0.13 \\
\text { (Cl 0.02-0.98) }\end{array}$ & & IAP: $7 \%$ \\
\hline \multirow[t]{2}{*}{ Bacteriodetes } & $\begin{array}{l}\text { Aloisio } 2016 \\
{[31]^{a}}\end{array}$ & $\begin{array}{l}\text { IAP } n=10 \\
\text { Control } \\
n=10\end{array}$ & & & & $\begin{array}{l}\text { IAP: } 16 \% \\
\text { Control: } \\
47.7 \% \\
p<0.05\end{array}$ & & \\
\hline & $\begin{array}{l}\text { Mazzola } 2016 \\
{[57]^{a}}\end{array}$ & $\begin{array}{l}\text { Mixed-fed IAP } \\
n=6 \\
\text { Mixed-fed } \\
\text { Control } n=6\end{array}$ & & & & $\begin{array}{l}\text { IAP: } 21 \% \\
\text { Control: } 36 \% \\
\text { RR } 0.59 \\
\text { (Cl } 0.3-0.93 \text { ) }\end{array}$ & & $\begin{array}{l}\text { IAP: } 34 \% \\
\text { Control: } 26 \% \\
\text { RR } 1.31 \text { (Cl } 0.85-2.01)\end{array}$ \\
\hline \multirow[t]{4}{*}{ Proteobacteria } & $\begin{array}{l}\text { Aloisio } 2016 \\
{[31]^{a}}\end{array}$ & $\begin{array}{l}\text { IAP } n=10 \\
\text { Control } n=10\end{array}$ & & & & $\begin{array}{l}\text { IAP: } 54.7 \% \\
\text { Control: } \\
15.5 \% \\
p<0.05\end{array}$ & & \\
\hline & $\begin{array}{l}\text { Arboleya } 2016 \\
\text { [33] }\end{array}$ & $\begin{array}{l}\text { IAP } n=14 \\
\text { Control } n=13\end{array}$ & & & & & & $\begin{array}{l}\text { Higher \% in IAP, } \\
p<0.001\end{array}$ \\
\hline & $\begin{array}{l}\text { Mazzola } 2016 \\
{[57]^{a}}\end{array}$ & $\begin{array}{l}\text { Breast-fed IAP } n=7 \\
\text { Breast-fed } \\
\text { Control } n=7\end{array}$ & & & & $\begin{array}{l}\text { Higher } \% \\
\text { in IAP, } \\
p<0.062\end{array}$ & & \\
\hline & & $\begin{array}{l}\text { Mixed-fed IAP } \\
n=6 \\
\text { Mixed-fed } \\
\text { Control } n=6\end{array}$ & & & & $\begin{array}{l}\text { IAP: } 37 \% \\
\text { Control: } 17 \% \\
\text { RR } 2.18 \\
\text { (CI } 1.32-3.60 \text { ) }\end{array}$ & & IAP: $28 \%$ \\
\hline \multirow[t]{2}{*}{ Firmicutes } & $\begin{array}{l}\text { Arboleya } 2016 \\
\text { [33] }\end{array}$ & $\begin{array}{l}\text { IAP } n=14 \\
\text { Control } n=13\end{array}$ & & & & & & $\begin{array}{l}\text { Lower } \% \text { in IAP, } \\
p<0.01\end{array}$ \\
\hline & $\begin{array}{l}\text { Mazzola } 2016 \\
{[57]^{a}}\end{array}$ & $\begin{array}{l}\text { Mixed-fed IAP } \\
n=6 \\
\text { Mixed-fed } \\
\text { Control } n=6\end{array}$ & & & & $\begin{array}{l}\text { IAP: } 41 \% \\
\text { Control: } 29 \% \\
\text { RR 1.14 } \\
\text { (Cl 0.96-2.08) }\end{array}$ & & IAP: $30 \%$ \\
\hline \multicolumn{9}{|l|}{ Family } \\
\hline \multirow[t]{2}{*}{ Bifidobacteriaceae } & $\begin{array}{l}\text { Aloisio } 2016 \\
{[31]^{a}}\end{array}$ & $\begin{array}{l}\text { IAP } n=10 \\
\text { Control } n=10\end{array}$ & & & & $\begin{array}{l}\text { IAP: } 0.02 \% \\
\text { Control: } \\
6.47 \% \\
p<0.05\end{array}$ & & \\
\hline & $\begin{array}{l}\text { Arboleya } 2015 \\
\text { [32] }\end{array}$ & $\begin{array}{l}\text { IAP } n=14 \\
\text { Control } n=13\end{array}$ & & & & & & $\begin{array}{l}\text { Lower \% in IAP, } \\
p<0.05\end{array}$ \\
\hline Comamonadaceae & $\begin{array}{l}\text { Arboleya } 2015 \\
{[32]}\end{array}$ & $\begin{array}{l}\text { IAP } n=14 \\
\text { Control } n=13\end{array}$ & & & & & & $\begin{array}{l}\text { Lower } \% \text { in IAP, } \\
p<0.05\end{array}$ \\
\hline
\end{tabular}


Table 1 Qualitative gut microbiota composition of IAP-treated and untreated infants (Continued)

\begin{tabular}{|c|c|c|c|c|c|c|c|}
\hline & $\begin{array}{l}\text { Arboleya } 2015 \\
\text { [32] }\end{array}$ & Control $n=13$ & & & & & $\begin{array}{l}\text { Higher \% in IAP, } \\
p<0.05\end{array}$ \\
\hline & $\begin{array}{l}\text { Mazzola } 2016 \\
{[57]^{a}}\end{array}$ & $\begin{array}{l}\text { Breast-fed IAP } n=7 \\
\text { Breast-fed } \\
\text { Control } n=7\end{array}$ & & & $\begin{array}{l}\text { Higher } \% \\
\text { in IAP, } \\
p=0.044\end{array}$ & & $\begin{array}{l}\text { IAP: } 44 \% \\
\text { Control: } 16 \% \\
\text { RR } 2.75 \text { (CI 1.67-4.54) }\end{array}$ \\
\hline & & $\begin{array}{l}\text { Mixed-fed IAP } n=6 \\
\text { Mixed-fed } \\
\text { Control } n=6\end{array}$ & & & $\begin{array}{l}\text { IAP: } 35 \% \\
\text { Control: } 17 \% \\
\text { RR } 2.06 \\
\text { (Cl 1.24-3.42) }\end{array}$ & & IAP: $28 \%$ \\
\hline & $\begin{array}{l}\text { Jaureguy } 2004 \\
{[39]^{\mathrm{a}}}\end{array}$ & $\begin{array}{l}\text { IAP } n=25 \\
\text { Control } n=25\end{array}$ & & $\begin{array}{l}\text { IAP } n=13 \\
\text { Control } \\
n=16 \\
p=0.58\end{array}$ & IAP: $0 \%$ & & \\
\hline Lachnospiraceae & $\begin{array}{l}\text { Mazzola } 2016 \\
{[57]^{a}}\end{array}$ & $\begin{array}{l}\text { IAP } n=14 \\
\text { Control } n=13\end{array}$ & & & & & IAP: $4 \%$ \\
\hline Leuconostaceae & $\begin{array}{l}\text { Arboleya } 2015 \\
\text { [32] }\end{array}$ & $\begin{array}{l}\text { IAP } n=14 \\
\text { Control } n=13\end{array}$ & $\begin{array}{l}\text { Lower } \% \text { in } \\
\text { IAP, } p<0.05\end{array}$ & & & & \\
\hline Micrococcaceae & $\begin{array}{l}\text { Arboleya } 2015 \\
\text { [32] }\end{array}$ & $\begin{array}{l}\text { IAP } n=14 \\
\text { Control } n=13\end{array}$ & & & & $\begin{array}{l}\text { Lower \% in } \\
\text { IAP, } p<0.05\end{array}$ & \\
\hline Propionibacteriaceae & $\begin{array}{l}\text { Arboleya } 2015 \\
\text { [32] }\end{array}$ & $\begin{array}{l}\text { IAP } n=14 \\
\text { Control } n=13\end{array}$ & & & & $\begin{array}{l}\text { Lower \% in } \\
\text { IAP, } p<0.05\end{array}$ & \\
\hline Staphylococcaceae & $\begin{array}{l}\text { Arboleya } 2015 \\
\text { [32] }\end{array}$ & $\begin{array}{l}\text { IAP } n=14 \\
\text { Control } n=13\end{array}$ & & & & & $\begin{array}{l}\text { Lower } \% \text { in IAP, } \\
p<0.05\end{array}$ \\
\hline Streptococcaceae & $\begin{array}{l}\text { Arboleya } 2015 \\
\text { [32] }\end{array}$ & $\begin{array}{l}\text { IAP } n=14 \\
\text { Control } n=13\end{array}$ & & & & & $\begin{array}{l}\text { Lower \% in IAP, } \\
p<0.05\end{array}$ \\
\hline Veillonellaceae & $\begin{array}{l}\text { Mazzola } 2016 \\
{[57]^{a}}\end{array}$ & $\begin{array}{l}\text { Breast-fed IAP } n=7 \\
\text { Breast-fed } \\
\text { Control } n=7\end{array}$ & & & & & $\begin{array}{l}\text { Lower } \% \text { in IAP, } \\
p=0.035\end{array}$ \\
\hline $\begin{array}{l}\text { Unclassified } \\
\text { Actinobacteria }\end{array}$ & $\begin{array}{l}\text { Arboleya } 2015 \\
\text { [32] }\end{array}$ & $\begin{array}{l}\text { IAP } n=14 \\
\text { Control } n=13\end{array}$ & & & & & $\begin{array}{l}\text { Lower \% in IAP, } \\
p<0.05\end{array}$ \\
\hline Unclassified Bacilli & & $\begin{array}{l}\text { IAP } n=14 \\
\text { Control } n=13\end{array}$ & & & & & $\begin{array}{l}\text { Lower } \% \text { in IAP, } \\
p<0.05\end{array}$ \\
\hline $\begin{array}{l}\text { Unclassified } \\
\text { Lactobacillales }\end{array}$ & & $\begin{array}{l}\text { IAP } n=14 \\
\text { Control } n=13\end{array}$ & & & & & $\begin{array}{l}\text { Lower } \% \text { in IAP, } \\
p<0.05\end{array}$ \\
\hline \multicolumn{8}{|l|}{ Genera } \\
\hline \multirow[t]{3}{*}{ Bacteroides } & $\begin{array}{l}\text { Jaureguy } 2004 \\
{[39]^{a}}\end{array}$ & $\begin{array}{l}\text { IAP } n=25 \\
\text { Control } n=25\end{array}$ & & $\begin{array}{l}\text { IAP } n=13 \\
\text { Control } \\
n=7 \\
p=0.15\end{array}$ & & & \\
\hline & $\begin{array}{l}\text { Mazzola } 2016 \\
{[57]^{a}}\end{array}$ & $\begin{array}{l}\text { Breast-fed IAP } n=7 \\
\text { Breast-fed } \\
\text { Control } n=7\end{array}$ & & & $\begin{array}{l}\text { IAP: } 7 \% \\
\text { Control: } 20 \% \\
p=0.078\end{array}$ & & \\
\hline & & $\begin{array}{l}\text { Mixed-fed IAP } n=6 \\
\text { Mixed-fed } \\
\text { Control } n=6\end{array}$ & & & $\begin{array}{l}\text { IAP: } 13 \% \\
\text { Control: } 32 \% \\
\text { RR } 0.41 \\
\text { (Cl } 0.23-0.73 \text { ) }\end{array}$ & & \\
\hline \multirow[t]{3}{*}{ Bifidobacteria } & $\begin{array}{l}\text { Jaureguy } 2004 \\
\text { [39] }^{a}\end{array}$ & $\begin{array}{l}\text { IAP } n=25 \\
\text { Control } n=25\end{array}$ & & $\begin{array}{l}\text { IAP } n=6 \\
\text { Control } \\
n=12 \\
p=0.18\end{array}$ & & & \\
\hline & $\begin{array}{l}\text { Mazzola } 2016 \\
{[57]^{a}}\end{array}$ & $\begin{array}{l}\text { Breast-fed IAP } n=7 \\
\text { Breast-fed } \\
\text { Control } n=7\end{array}$ & & & $\begin{array}{l}\text { IAP: } 0 \% \\
\text { Control: } 16 \% \\
p=0.001\end{array}$ & & $\begin{array}{l}\text { IAP: } 6 \% \text { (compared } \\
\text { to day } 7, p=0.025 \text { ) } \\
\text { Control: } 6 \%\end{array}$ \\
\hline & $\begin{array}{l}\text { Mazzola } 2016 \\
{[57]^{a}}\end{array}$ & $\begin{array}{l}\text { Mixed-fed IAP } n=6 \\
\text { Mixed-fed } \\
\text { Control } n=6\end{array}$ & & & $\begin{array}{l}\text { IAP: } 1 \% \text { or } 0 \% \\
\text { Control: } 5 \%\end{array}$ & & $\begin{array}{l}\text { IAP: } 6 \% \text { (compared } \\
\text { to day } 7, p=0.013) \\
\text { Control: } 19 \% \\
\text { RR: } 0.32 \text { (Cl } 0.13-0.76)\end{array}$ \\
\hline
\end{tabular}


Table 1 Qualitative gut microbiota composition of IAP-treated and untreated infants (Continued)

\begin{tabular}{|c|c|c|c|c|c|}
\hline Clostridia & $\begin{array}{l}\text { Jaureguy } 2004 \\
{[39]^{\mathrm{a}}}\end{array}$ & $\begin{array}{l}\text { IAP } n=25 \\
\text { Control } n=25\end{array}$ & $\begin{array}{l}\text { IAP } n=3 \\
\text { Control } \\
n=10 \\
p=0.04\end{array}$ & & \\
\hline Enterococci & $\begin{array}{l}\text { Jaureguy } 2004 \\
\text { [39] }^{\mathrm{a}}\end{array}$ & $\begin{array}{l}\text { IAP } n=25 \\
\text { Control } n=25\end{array}$ & $\begin{array}{l}\text { IAP } n=15 \\
\text { Control } \\
n=17 \\
p=0.73\end{array}$ & & \\
\hline Escherichia & $\begin{array}{l}\text { Mazzola } 2016 \\
{[57]^{a}}\end{array}$ & $\begin{array}{l}\text { Breast-fed IAP } n=7 \\
\text { Breast-fed } \\
\text { Control } n=7\end{array}$ & & $\begin{array}{l}\text { IAP: } 52 \% \\
\text { Control: } 14 \% \\
\text { RR 3.71 } \\
\text { (Cl } 2.21-6.25)\end{array}$ & \\
\hline Staphylococci & $\begin{array}{l}\text { Jaureguy } 2004 \\
\text { [39] }^{\mathrm{a}}\end{array}$ & $\begin{array}{l}\text { IAP } n=25 \\
\text { Control } n=25\end{array}$ & $\begin{array}{l}\text { IAP } n=21 \\
\text { Control } \\
n=22 \\
p=1.00\end{array}$ & & \\
\hline Streptococci & $\begin{array}{l}\text { Mazzola } 2016 \\
{[57]^{a}}\end{array}$ & $\begin{array}{l}\text { Mixed-fed IAP } n=6 \\
\text { Mixed-fed } \\
\text { Control } n=6\end{array}$ & & $\begin{array}{l}\text { IAP: } 32 \% \\
\text { Control: } 10 \% \\
\text { RR 3.2 } \\
\text { (CI 1.66-6.15) }\end{array}$ & $\begin{array}{l}\text { IAP: } 8 \% \text { (compared } \\
\text { to day } 7, p=0.042 \text { ) }\end{array}$ \\
\hline $\begin{array}{l}\text { Other microbial } \\
\text { genus }\end{array}$ & $\begin{array}{l}\text { Aloisio } 2016 \\
{[31]^{a}}\end{array}$ & $\begin{array}{l}\text { IAP } n=10 \\
\text { Control } n=10\end{array}$ & & $\begin{array}{l}\text { No significant } \\
\text { differences }\end{array}$ & \\
\hline
\end{tabular}

$\mathrm{Cl}$ confidence interval, IAP intrapartum antibiotic prophylaxis, $p$ probability value, RR risk ratio

Numbers in italics calculated by reviewers

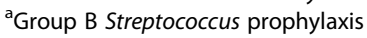

compared to no amoxicillin-clavulanate for preterm labour. The risk of cerebral palsy was higher in infants whose mothers received any erythromycin versus no erythromycin (placebo or amoxicillin-clavulanate) (53/1611 [3\%] and 27/ 1562 [2\%] OR $=1.93$ 95\% CI 1.21-3.09) or any amoxicillinclavulanate versus no amoxicillin-clavulanate (placebo or erythromycin) (50/1587 [3\%] and 30/1586 [2\%] OR = 1.69 95\% CI 1.07-2.67). More children who developed cerebral palsy had been born to mothers who had received both antibiotics (35/735) than to mothers who received erythromycin only (18/785), amoxicillin-clavulanate only (15/763), or double placebo (12/735) (both drugs versus double placebo: $\mathrm{OR}=2.9195 \%$ CI 1.50-5.65). The authors also found that any erythromycin significantly increased the risk of bowel problems (64/1611 [4\%] versus 38/1562 [2\%] $\mathrm{OR}=1.6695 \%$ CI 1.10-2.49) and functional impairment $(658 / 1554$ [42\%] versus 574/1498 [38\%] OR $=1.18$ 95\% CI $1.02-1.37)$ compared to no erythromycin. None of these effects were found for either erythromycin or amoxicillinclavulanate alone compared to placebo, however this may have been a result of insufficient power.

The study had a low risk of bias in all major domains, however there were critical limitations. Multiple statistical comparisons were conducted on a relatively small sample size increasing the probability of getting a significant effect due to chance. In addition to cerebral palsy, functional impairment, and bowel problems, the authors also investigated diabetes, behavioural problems, educational attainment, attention deficit hyperactivity disorder, and other developmental problems, and did not find any significant differences between any of the treatment and control groups. This is particularly important as the biological plausibility of IAP increasing the risk of cerebral palsy is unknown.

\section{Discussion}

In this systematic review, we presented the evidence on adverse events experienced by the mother and/or her child after treatment with intrapartum antibiotic prophylaxis (IAP). Despite a wide range of adverse outcomes reported from 17 observational studies and 13 RCTs, there was limited high quality information to determine the frequency of adverse events from IAP for neonatal GBS disease prevention. The evidence contains much uncertainty, with a substantial evidence gap around the long-term effects of IAP. The only RCT investigating the long-term effects of IAP reported a moderate effect of severe consequences such as cerebral palsy. This trial had limited applicability as it used a different drug, a longer drug regimen, and pre-term rather than term labour compared to IAP for GBS prevention. We also found consistent observational evidence that IAP for neonatal GBS prevention alters the infant microbiome, with some studies showing changes up to 90 days of life. However, these study populations were not followed through to clinical outcomes, therefore the short- and long-term clinical significance of the changes are unknown. Finally, there was evidence for increased antibiotic resistance in some, but not all studies, with no 


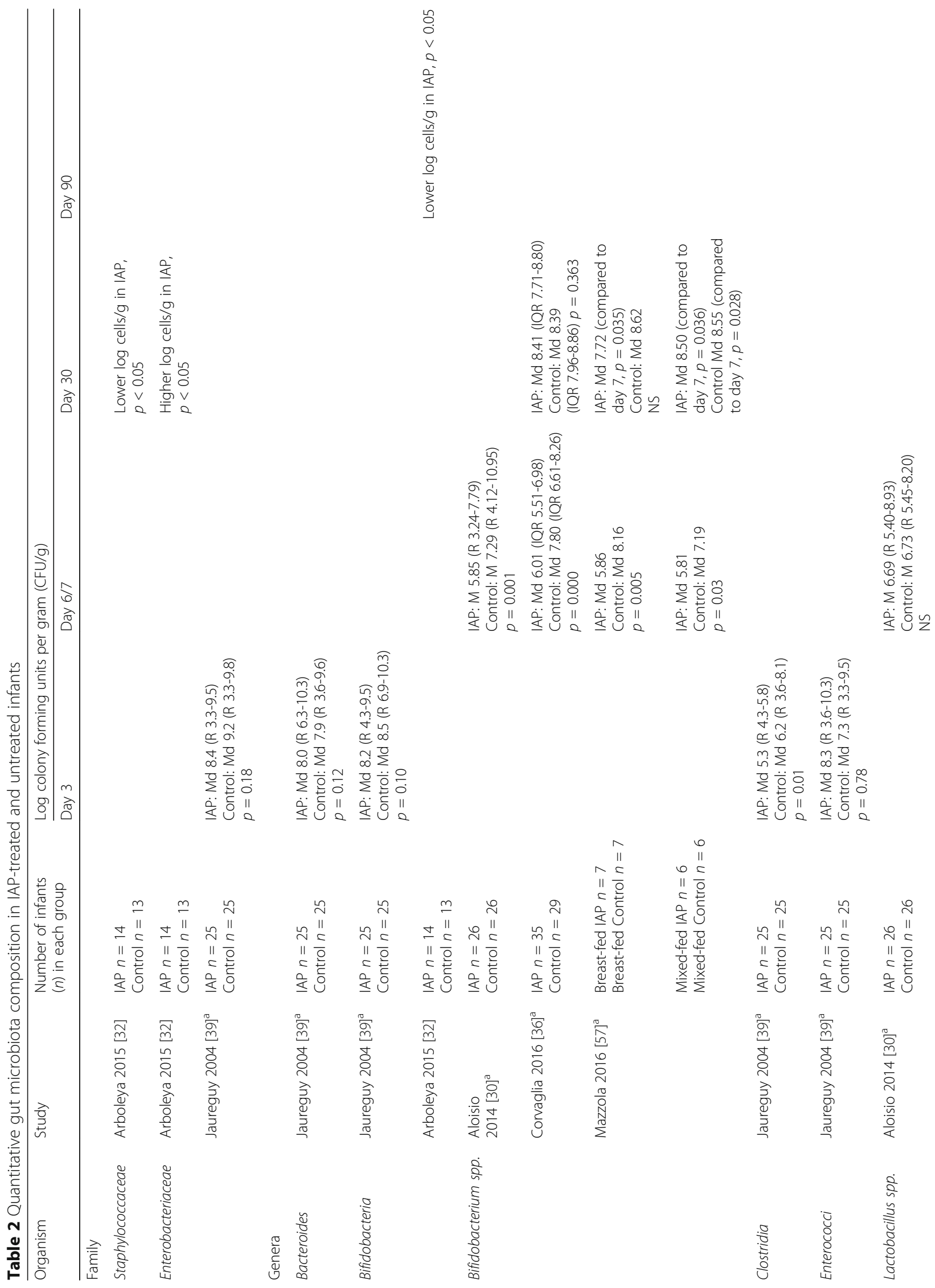




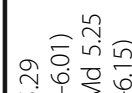

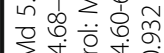

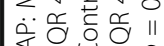

-

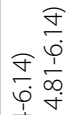

先

鱼

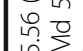

ఫิ

定

ข

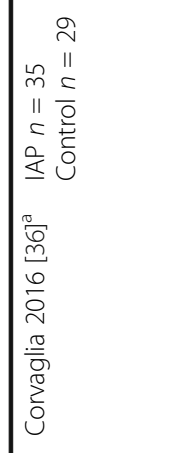

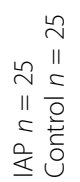

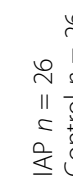

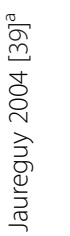

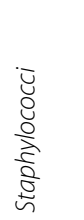

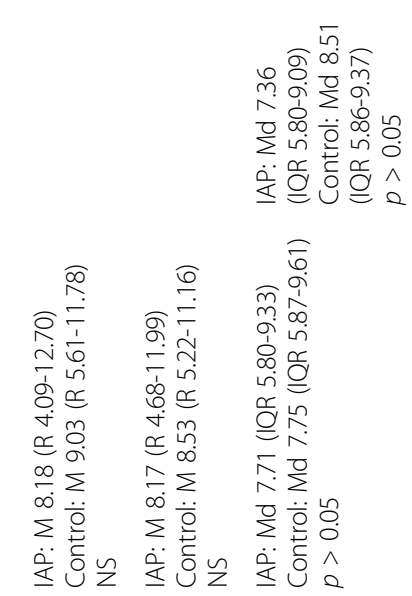

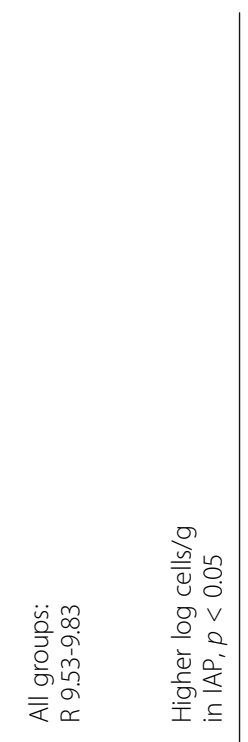

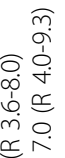

능물

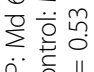

过

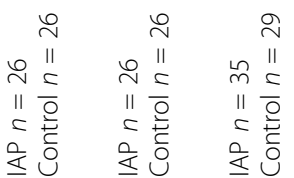

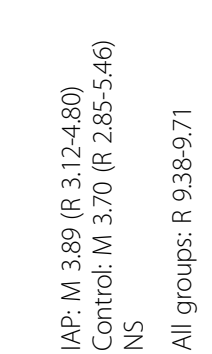


evidence of a reduction. However, this observational evidence was at high or unclear risk of bias due to confounding variables.

Our review is the first systematic assessment of the literature on the adverse events from IAP. We had an extensive search with no date limit, and referencing checking of all included papers and relevant systematic reviews. We also had expert input, two reviewers conducting processes, including quality appraisal using validated tools, and we calculated summary measures for study outcomes where they were not available. However, as our search was broad and focused heavily on harms or adverse events search terms, we may not have found studies which investigated an outcome that would potentially be an adverse event, but may not have been indexed as such. Furthermore, as we only included studies for which full texts were available in English, adverse events reported in other languages may have been missed. We were also unable to conduct any metaanalyses due to the heterogeneity across the adverse events investigated.

Previous literature has suggested that initial bacterial colonisation of the gut plays an important role in the development of the mucosal immune system of infants [21-23, 42]. Microbiota changes from antibiotics have been associated with respiratory problems in children such as asthma, metabolic problems such as obesity and diabetes, and autism [21-23]. For example, Cox et al. (2014) demonstrated that low dose penicillin delivered after birth caused gut microbiota changes that led to permanent abnormalities in metabolism and immunity in mice [60]. More recently, antibiotic exposure before six months of age or repeatedly during infancy was associated with increased body mass and height in healthy children [23]. The antibiotics in these studies were not administered intrapartum. Long-term follow-up investigations linking antibiotic prophylaxis specifically during labour and early microbiota alterations to clinical consequences are required to understand their significance.

Evidence on the increase of antibiotic resistance after IAP was inconsistent. Literature on the trends of antibiotic resistance in countries offering IAP for neonatal GBS prevention has shown an increase in rates overtime. Resistance to clindamycin and erythromycin has increased in the last 20 years [9], with reported resistance to erythromycin at 30\% or higher in the US and Switzerland, and above $15 \%$ for clindamycin in the US, Switzerland, and England [61-63]. Similarly, although GBS remains almost universally susceptible to penicillin [16], in 2005 in the US, $0.2 \%$ of GBS isolates had reached the upper level of susceptibility $[61,64]$. However, these trends are difficult to attribute specifically to IAP for GBS prevention, and could be due to other factors.
Kenyon et al.'s (2008) RCT had a low risk of bias and showed that IAP was associated with an increase in the severe consequences such as cerebral palsy [41]. However, the effect size was small, and with multiple statistical comparisons conducted on the same population, the probability of a chance result is increased. Based on previous literature the plausible biological mechanisms through which IAP may cause the development of cerebral palsy are unknown $[41,65]$. Complicating these findings, a second trial on IAP for pregnant women with preterm rupture of the membranes (excluded due to signs of infection confounding IAP effects) found no difference in the proportion of children with cerebral palsy between treated or untreated women [66]. Therefore, why cerebral palsy occurred in the first study and whether it would occur as a result of use of IAP for neonatal GBS prevention, which involves different drug regimens and durations, is uncertain.

A Cochrane meta-analysis concluded that despite an 83\% reduction in EOGBS incidence from IAP, IAP for maternal GBS is not supported by conclusive evidence due to a high risk of bias across RCTs [67]. Combining this uncertainty with the results of this review makes it increasingly difficult to ascertain whether the benefits of administering IAP for EOGBS prevention outweigh the harms to mothers and children. Large, well-designed RCTs required to answer this question may no longer be feasible as IAP is now the recommended treatment. Instead, large, better quality, and longitudinal observational studies across countries with widespread IAP may be an alternative to understand the adverse events occurring in participants treated with IAP. Expanding EOGBS prevention from risk-based strategies to universal antenatal screening introduces the risk of increasing the number of low risk women treated with IAP. Up to $30 \%$ of mothers positive in pregnancy may become negative by birth, and less than $1 \%$ of mothers colonised in labour have a baby with EOGBS, all of whom could be unnecessarily exposed to potential harms [13, 7]. As observational evidence on universal GBS screening effectiveness is limited due to inherent biases $[68,9]$, an $\mathrm{RCT}$ could inform on both the effectiveness and harms of screening and IAP treatment for neonatal GBS disease prevention.

\section{Conclusions}

The evidence on the adverse events from IAP treatment for neonatal GBS disease prevention is unclear, inconsistent, and/or at risk of bias. There is consistent evidence that GBS antibiotic prophylaxis alters the infant microbiome, and some inconsistent evidence that IAP increases antibiotic resistance. However, this evidence is at risk of bias, and the clinical consequences of the microbiome alterations are unknown. There is evidence 
from a single long-term RCT associating IAP in preterm labour with potentially severe consequences such as cerebral palsy, however, it has applicability concerns, unclear biological plausibility, and was not replicated in a similar RCT. These limitations preclude the drawing of any accurate conclusions on the frequency of adverse events from IAP treatment for neonatal GBS disease prevention. Larger, better quality, and longer studies are needed to provide estimates of adverse events from IAP treatment for neonatal GBS disease prevention.

\section{Additional file}

Additional file 1: The Additional file contains three items. The first shows the search strategy used for Medline that was adapted for other databases; the second lists the studies that were excluded $(n=227)$ at the full text stage with the reasons for their exclusion; and the third is Table S1. that summarises all of the included studies, their characteristics, and their findings. (DOCX $225 \mathrm{~kb}$ )

\section{Abbreviations}

CFU: Colony forming units; Cl: Confidence intervals; E. coli: Escherichia coli; EOGBS: Early-onset neonatal group B Streptococcus disease; GBS: Group B Streptococcus; IAP: Intrapartum antibiotic prophylaxis; OR: Odds ratio; RCT: Randomised controlled trial; RD: Risk difference; RoB: Risk of Bias; RoBANS: Risk of bias assessment tool for nonrandomised studies; RR: Relative risk; S. aureus: Staphylococcus aureus; S. pneumoniae: Streptococcus pneumoniae; UK: United Kingdom; US: United States of America

\section{Acknowledgements}

We would like to thank Dr. Michael Millar, Dr. Arlene Reynolds, and Dr. Magdalena Skrybant for providing advice and input into this research and our funders, the UK National Screening Committee and the National Institute of Health Research.

\section{Funding}

This work was funded by UK National Screening Committee (NSC). Sian Taylor-Phillips, Aileen Clarke, Chris Stinton, and Hannah Fraser are supported by the National Institute of Health Research (NIHR) Collaboration for Leadership in Applied Health Research and Care West Midlands. The funders had no role in the study design, collection, analysis and interpretation of data, writing of the report, and in the decision to submit the article for publication.

\section{Availability of data and materials}

Not applicable.

\section{Authors' contributions}

FS secured funding, co-ordinated the review process, developed the protocol, created and applied the search strategy to collect the data, sifted, extracted, quality assessed, and synthesised the data and wrote the report. CS, BT, JP and JG carried out the second review of the article selection and reviewed the paper for redrafting and conducted data extraction and quality assessment, and CS and BT contributed to protocol development. BT provided obstetrics and gynaecology expertise. KF contributed to protocol development, carried out data extraction, quality assessment, and reviewed the paper for redrafting. OU contributed to methods for protocol development and reviewed the paper for redrafting. NM, ER and CB contributed to protocol development and report writing, providing expertise on infection and microbiology. SJ and HF contributed to protocol development search strategy development, accessing papers, and reviewed the report for redrafting. AC contributed to protocol development, and reviewed the paper for redrafting. STP secured the funding, co-ordinated the review process, developed the protocol, and wrote the report. All authors read and approved the final manuscript.

\section{Authors' information}

FS is an experienced systematic reviewer, specialising in screening and infectious diseases. She is currently leading research using international data to understand the benefits and harms of GBS screening, leading the Cochrane systematic review on tests for maternal GBS carriage, has led a UK NSC review on international screening policy, and contributed to a health technology assessment for NICE. Dr. Julia Geppert, Dr. Chris Stinton, Dr. Jacoby Patterson, and Karoline Freeman are expert systematic reviewers specialising in screening and test accuracy, and have conducted health technology assessments for NICE and UK NSC policy reviews.

Dr. Tan is a consultant obstetrician \& gynaecologist practising in the UK for 17 years, and has managed numerous patients with GBS in pregnancy. He is a Royal College of Obstetricians \& Gynaecologists accredited subspecialist in Reproductive Medicine, a Member of the Royal College of Obstetricians \& Gynaecologists (MRCOG) and Fellow of the Royal College of Surgeons (FRCS).

Professor Noel McCarthy is an academic public health physician and epidemiologist with qualifications including MPH, MSC (Medical Statistics) and DPhil (Epidemiology). He has over 60 peer-reviewed publications on public health issues in infectious disease control. Dr. Esther Robinson is the Lead Public Health Microbiologist for East Midlands Pubic Health England. She has a DPhil in Clinical Laboratory Sciences, and has experience with infection and antibiotic resistance. She is a fellow of the Royal College of Pathologists (FRCPath), member of the Royal College of Physicians (MRCP(UK)). Dr. Colin Brown is a Consultant in Infectious Diseases and Medical Microbiology at Public Health England with expertise in clinical infectious disease and disease policy.

Dr. Olalekan Uthman has worked across a wide range of health technology a focus on meta-analytical research and infectious diseases. He is an experienced Cochrane author and Cochrane Infectious Disease Group Editor and has contributed to several recent UK NICE public health and intervention guidelines and World Health Organization policies.

Samantha Johnson is an academic support librarian specialising in medicine and life sciences. Hannah Fraser has a Bachelor in Science, has studied the Masters in Screening course, and has provided administrative support to UK NSC policy reviews for health screening programmes.

Professor Aileen Clarke is a clinical public health academic who heads the Division of Health Sciences. She is a Fellow of the Higher Education Academy and a fellow of the Royal College of General Practitioners, has over 99 publications on evidence for clinical practice and policy and health technology assessment and has active links with national and international bodies such as the Nuffield Trust, the Faculty of Public Health Research Policy Committee, and the Society for Medical Decision Making. Professor Clarke leads one of nine Technology Assessment Review teams providing systematic reviews to NICE.

Dr. Sian Taylor-Phillips is an Associate Professor of Screening and Test Evaluation with wide experience in systematic reviews and cost effectiveness analysis specialising in population screening. She is well published in academic papers on population screening, and has led research in a range of screening programmes, including leading UK NSC policy reviews. She has also provided oral evidence to the Science and Technology Parliamentary Committee on Health Screening.

Ethics approval and consent to participate

Not applicable.

\section{Consent for publication}

Not applicable.

\section{Competing interests}

The authors declare that they have no competing interests.

\section{Publisher's Note}

Springer Nature remains neutral with regard to jurisdictional claims in published maps and institutional affiliations.

\section{Author details}

'Division of Health Sciences, University of Warwick Medical School, Gibbet Hill Campus, Coventry CV4 7AL, UK. '2Department of Obstetrics and Gynaecology, Birmingham Heartlands Hospital, Heart of England NHS Foundation Trust, Birmingham B9 5SS, UK. ${ }^{3}$ Birmingham Public Health 
Laboratory (PHE), Heartlands Hospital, Birmingham B9 5SS, UK. ${ }^{4}$ Bacteria Reference Department, National Infection Service, Public Health England, 61 Colindale Ave, London NW95EQ, UK.

Received: 28 February 2017 Accepted: 18 July 2017

Published online: 26 July 2017

\section{References}

1. Edwards M, Baker C. Group B streptococcal infections. In:. In: Remington J, Klein J, editors. Infectious diseases of the fetus and newborn infant. Philadelphia: Saunders; 2001. p. 1091-1156.

2. Daniels JP, Gray J, Pattison HM, Gray R, Hills RK, Khan KS. Intrapartum tests for group B streptococcus: accuracy and acceptability of screening. BJOG. 2011;118(2):257-65. doi:10.1111/j.1471-0528.2010.02725.x.

3. Regan JA, Klebanoff MA, Nugent RP. The epidemiology of group $B$ streptococcal colonization in pregnancy. Vaginal infections and prematurity study group. Obstet Gynecol. 1991;77(4):604-10.

4. Barcaite E, Bartusevicius A, Tameliene R, Kliucinskas M, Maleckiene L, Nadisauskiene R. Prevalence of maternal group B streptococcal colonisation in European countries. Acta Obstet Gynecol Scand. 2008;87:260-71.

5. Kwatra G, Cunnington MC, Merrall E, Adrian PV, Ip M, Klugman KP, et al. Prevalence of maternal colonisation with group B streptococcus: a systematic review and meta-analysis. Lancet Infect Dis. 2016;16(9):1076-84. doi:10.1016/s1473-3099(16)30055-x.

6. Colbourn T, Gilbert R. An overview of the natural history of early onset group B streptococcal disease in the UK. Early Hum Dev. 2007:83:149-56.

7. Le Doare K, Heath PT. An overview of global GBS epidemiology. Vaccine. 2013;31(Suppl 4):D7-12. doi:10.1016/j.vaccine.2013.01.009.

8. Edmond K, Kortsalioudaki C, Scott S, Schrag S, Zaidi A, Cousens S, et al. Group B streptococcal disease in infants aged younger than 3 months: systematic review and meta-analysis. Lancet. 2012;379:547-56.

9. Verani JR, McGee L, Schrag SJ. Prevention of perinatal group B streptococcal disease-revised guidelines from CDC, 2010. MMWR Recomm Rep. 2010:59(RR-10):1-36.

10. Royal College of Obstetricians and Gynaecologists. Prevention of early onset neonatal group B streptococcal disease. Green-top guideline no. 36. 2nd ed. United Kingdom: RCOG; 2012.

11. Di Renzo GC, Melin P, Berardi A, Blennow M, Carbonell-Estrany X, Donzelli GP, et al. Intrapartum GBS screening and antibiotic prophylaxis: a European consensus conference. J Matern Fetal Neonatal Med. 2015;28(7):766-82. doi:10.3109/14767058.2014.934804.

12. Rodriguez-Granger J, Alvargonzalez JC, Berardi A, Berner R, Kunze M, Hufnagel $M$, et al. Prevention of group B streptococcal neonatal disease revisited. The DEVANI European project. Eur J Clin Microbiol Infect Dis. 2012;31(9):2097-104. doi:10.1007/s10096-012-1559-0.

13. Valkenburg-van den Berg AW, Houtman-Roelofsen RL, Oostvogel PM, Dekker FW, Dorr PJ, Sprij AJ. Timing of group B streptococcus screening in pregnancy: a systematic review. Gynecol Obstet Investig. 2010;69(3):174-83. doi:10.1159/000265942.

14. UK National Screening Committee. Screening for group B streptococcal infection in pregnancy: external review against programme appraisal criteria for the UK National Screening Committee (UK NSC). United Kingdom: UK NSC2012.

15. Colbourn T, Asseburg C, Bojke L, Philips Z, Claxton K, Ades AE, et al. Prenatal screening and treatment strategies to prevent group $B$ streptococcal and other bacterial infections in early infancy: cost-effectiveness and expected value of information analyses. Health technology assessment (Winchester, England). 2007;11(29):1-226, iii.

16. Schrag S, Gorwitz R, Fultz-Butts K, Schuchat A. Prevention of Perinatal group $B$ streptococcal disease. Revised Guidelines from CDC. MMWR. 2002;51(RR-11):1-23.

17. Towers CV, Carr MH, Padilla G, Asrat T. Potential consequences of widespread antepartal use of ampicillin. Am J Obstet Gynecol. 1998;179(4):879-83.

18. Terrone DA, Rinehart BK, Einstein MH, Britt LB, Martin JN Jr, Perry KG. Neonatal sepsis and death caused by resistant Escherichia Coli: possible consequences of extended maternal ampicillin administration. Am J Obstet Gynecol. 1999;180(6 Pt 1):1345-8.

19. Gilbert R. Prenatal screening for group b streptococcal infection: gaps in the evidence. Int J Epidemiol. 2003;33:2-8

20. Rouphael NG, O'Donnell JA, Bhatnagar J, Lewis F, Polgreen PM, Beekmann S, et al. Clostridium Difficile-associated diarrhea: an emerging threat to pregnant women. Am J Obstet Gynecol. 2008;198(6):635 e1-6. doi:10.1016/j.ajog.2008.01.062
21. Colbourn TE, Asseburg C, Bojke L, Philips Z, Welton NJ, Claxton K, et al. Preventive strategies for group B streptococcal and other bacterial infections in early infancy: cost effectiveness and value of information analyses. BMJ. 2007:335(7621):655. doi:10.1136/bmj.39325.681806.AD.

22. Azad MB, Konya T, Persaud RR, Guttman DS, Chari RS, Field CJ, et al. Impact of maternal intrapartum antibiotics, method of birth and breastfeeding on gut microbiota during the first year of life: a prospective cohort study. BJOG. 2015; doi:10.1111/1471-0528.13601.

23. Saari A, Virta L, Sankilampi U, Dunkel L, Saxen H. Antibiotic exposure in infancy and risk of being overweight in the first 24 months of life. Pediatrics. 2015:135(4):617-26. doi:10.1542/peds.2014-3407.

24. Moher D, Shamseer L, Clarke M, Ghersi D, Liberati A, Petticrew M, et al. Preferred reporting items for systematic review and meta-analysis protocols (PRISMA-P) 2015 statement. Syst Rev. 2015;4:1 doi:10.1186/2046-4053-4-1.

25. Golder S, Loke YK. Sensitivity and precision of adverse effects search filters in MEDLINE and EMBASE: a case study of fractures with thiazolidinediones. Health Inf Libr J. 2012;29(1):28-38. doi:10.1111/j.1471-1842.2011.00972.x.

26. Health Information Research Unit. Search Filters for MEDLINE in Ovid Syntax and the PubMed translation McMaster University, Ontario. http://hiru. mcmaster.ca/hiru/HIRU_Hedges_MEDLINE_Strategies.aspx. Accessed Mar 2016.

27. Daniels J, Gray J, Pattison H, Roberts T, Edwards E, Milner P, et al. Rapid testing for group B streptococcus during labour: a test accuracy study with evaluation of acceptability and cost-effectiveness. Health technology assessment (Winchester, England). 2009;13(42):1-154, iii-iv. doi:10.3310/hta13420.

28. Higgins JP, Altman DG, Gotzsche PC, Juni P, Moher D, Oxman AD, et al. The Cochrane Collaboration's tool for assessing risk of bias in randomised trials. BMJ. 2011;343:d5928. doi:10.1136/bmj.d5928.

29. Kim SY, Park JE, Lee YJ, Seo HJ, Sheen SS, Hahn S, et al. Testing a tool for assessing the risk of bias for nonrandomized studies showed moderate reliability and promising validity. J Clin Epidemiol. 2013;66(4):408-14 doi:10.1016/j.jclinepi.2012.09.016.

30. Aloisio I, Mazzola G, Corvaglia LT, Tonti G, Faldella G, Biavati B, et al. Influence of intrapartum antibiotic prophylaxis against group $B$ streptococcus on the early newborn gut composition and evaluation of the anti-streptococcus activity of Bifidobacterium strains. Appl Microbiol Biotechnol. 2014;98(13):6051-60.

31. Aloisio I, Quagliariello A, De Fanti S, Luiselli D, De Filippo C, Albanese D et al. Evaluation of the effects of intrapartum antibiotic prophylaxis on newborn intestinal microbiota using a sequencing approach targeted to multi hypervariable 165 rDNA regions. Appl Microbiol Biotechnol. 2016;100(12):5537-46. doi:http://dx.doi.org/10.1007/s00253-016-7410-2.

32. Arboleya S, Sanchez B, Milani C, Duranti S, Solis G, Fernandez N, et al. Intestinal microbiota development in preterm neonates and effect of Perinatal antibiotics. J Pediatr. 2015;166(3):538-44.

33. Arboleya S, Sanchez B, Solis G, Fernandez N, Suarez M, Hernandez-Barranco AM et al. Impact of Prematurity and Perinatal Antibiotics on the Developing Intestinal Microbiota: A Functional Inference Study. Int. 2016;17(5). doi:http:// dx.doi.org/10.3390/ijms17050649.

34. Ashkenazi-Hoffnung L, Melamed N, Ben-Haroush A, Livni G, Amir J, Bilavsky E. The Association of Intrapartum Antibiotic Exposure with the incidence and antibiotic resistance of infantile late-onset serious bacterial infections. Clin Pediatr. 2011;50(9):827-33

35. Briody VA, Albright CM, Has P, Hughes BL. Use of Cefazolin for group B streptococci prophylaxis in women reporting a penicillin allergy without anaphylaxis. Obstet Gynecol. 2016;127(3):577-83.

36. Corvaglia L, Tonti G, Martini S, Aceti A, Mazzola G, Aloisio I, et al. Influence of Intrapartum antibiotic prophylaxis for group B streptococcus on gut microbiota in the first month of life. J Pediatr Gastroenterol Nutr. 2016;62(2):304-8

37. Dinsmoor MJ, Viloria R, Lief L, Elder S. Use of intrapartum antibiotics and the incidence of postnatal maternal and neonatal yeast infections. Obstet Gynecol. 2005:106(1):19-22.

38. Glasgow TS, Young PC, Wallin J, Kwok C, Stoddard G, Firth S, et al. Association of intrapartum antibiotic exposure and late-onset serious bacterial infections in infants. Pediatrics. 2005;116(3):696-702.

39. Jaureguy F, Carton M, Panel P, Foucaud P, Butel MJ, Doucet-Populaire F. Effects of intrapartum penicillin prophylaxis on intestinal bacterial colonization in infants. J Clin Microbiol. 2004:42(11):5184-8.

40. Kampikaho A, Irwig LM. A randomized trial of penicillin and streptomycin in the prevention of post-partum infection in Uganda. Int J Gynaecol Obstet. 1993:41(1):43-52. 
41. Kenyon S, Pike K, Jones DR, Brocklehurst P, Marlow N, Salt A et al. Childhood outcomes after prescription of antibiotics to pregnant women with spontaneous preterm labour: 7-year follow-up of the ORACLE II trial. Lancet. 2008;372(9646):1319-1327. doi:http://dx.doi.org/10.1016/S0140-6736(08)61203-9.

42. Keski-Nisula L, Kyynarainen HR, Karkkainen U, Karhukorpi J, Heinonen S, Pekkanen J. Maternal intrapartum antibiotics and decreased vertical transmission of lactobacillus to neonates during birth. Acta Paediatr. 2013;102(5):480-5.

43. Nadisauskiene R, Bergstrom S. Impact of intrapartum intravenous ampicillin on pregnancy outcome in women with preterm labor: a randomised, placebo-controlled study. Gynecol Obstet Investig. 1996:41(2):85-8

44. Stoll BJ, Hansen N, Fanaroff AA, Wright LL, Carlo WA, Ehrenkranz RA et al. Changes in pathogens causing early-onset sepsis in very-low-birth-weight infants. N Engl J Med. 2002;347(4):240-7. doi:http://dx.doi.org/10.1056/NEJMoa012657.

45. Wohl DL, Curry WJ, Mauger D, Miller J, Tyrie K. Intrapartum antibiotics and childhood atopic dermatitis. J Am Board Fam Med. 2015:28(1):82-9. doi:http://dx.doi.org/10.3122/jabfm.2015.01.140017.

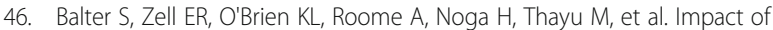
intrapartum antibiotics on the care and evaluation of the neonate. Pediatr Infect Dis J. 2003;22(10):853-7. doi:10.1097/01.inf.0000090920.22425.dc.

47. Cox SM, Bohman VR, Sherman ML, Leveno KJ. Randomized investigation of antimicrobials for the prevention of preterm birth. Am J Obstet Gynecol. 1996;174(1 Pt 1):206-10.

48. Gordon M, Samuels P, Shubert P, Johnson F, Gebauer C, lams J. A randomized, prospective study of adjunctive ceftizoxime in preterm labor. Am J Obstet Gynecol. 1995;172(5):1546-52.

49. Keettel WC, Plass ED. Prophylactic administration of penicillin to obstetric patients: additional data. J Am Med Assoc. 1950;142(5):324-8. doi:10.1001/ jama.1950.02910230026007

50. Keettel WC, Scott JW, Plass ED. An evaluation of prophylactic penicillin administration to parturient women. Am J Obstet Gynecol. 1949;58(2):335-44. doi:10.1016/0002-9378(49)90387-7.

51. Keuchkerian SE, Sosa CG, Fernandez A, Alonso JG, Laborde A Cuadro JC. Effect of amoxicillin sulbactam in threatened preterm labour with intact membranes: a randomised controlled trial. Eur J Obstet Gynecol Reprod Biol. 2005;119(1):21-6. doi:10.1016/j.ejogrb.2004.05.010.

52. McGregor JA, French Jl, Reller LB, Todd JK, Makowski EL. Adjunctive erythromycin treatment for idiopathic preterm labor: results of a randomized, double-blinded, placebo-controlled trial. Am J Obstet Gynecol. 1986;154(1):98-103.

53. Rajaei M, Sultani M, Zare S. A randomized controlled trial of adjunctive erythromycin in women with idiopathic preterm labor. J Matern Fetal Neonatal Med. 2006;19(1):17-20. doi:10.1080/14767050500361455.

54. Sinha A, Yokoe D, Platt R. Intrapartum antibiotics and neonatal invasive infections caused by organisms other than group B streptococcus. J Pediatr. 2003:142(5):492-7. doi:10.1067/mpd.2003.154.

55. Svare J, Langhoff-Roos J, Andersen LF, Kryger-Baggesen N, BorchChristensen $\mathrm{H}$, Heisterberg $\mathrm{L}$, et al. Ampicillin-metronidazole treatment in idiopathic preterm labour: a randomised controlled multicentre trial. Br J Obstet Gynaecol. 1997;104(8):892-7.

56. Lin FY, Troendle JF. Hypothesis: neonatal respiratory distress may be related to asymptomatic colonization with group B streptococci. Pediatr Infect Dis J. 2006;25(10):884-8

57. Mazzola G, Murphy K, Ross RP, Di Gioia D, Biavati B, Corvaglia LT et al. Early gut microbiota perturbations following intrapartum antibiotic prophylaxis to prevent group B streptococcal disease. PLoS One. 2016;11 (6) (no pagination)(e0157527). doi:http://dx.doi.org/10.1371/journal.pone.0157527.

58. Roca A, Oluwalana C, Bojang A, Camara B, Kampmann B, Bailey R et al. Oral azithromycin given during labour decreases bacterial carriage in the mothers and their offspring: a double-blind randomized trial. Clin Microbiol Infect. 2016;22(6). doi:10.1016/j.cmi.2016.03.005.

59. Salman S, Davis TM, Page-Sharp M, Camara B, Oluwalana C, Bojang A, et al. Pharmacokinetics of transfer of Azithromycin into the breast milk of African mothers. Antimicrob Agents Chemother. 2015;60(3):1592-9. doi:10.1128/aac.02668-15

60. Cox LM, Yamanishi S, Sohn J, Alekseyenko AV, Leung JM, Cho I. Altering the intestinal microbiota during a critical developmental window has lasting metabolic consequences. Cell. 2014;158:705-21.
61. Phares CR, Lynfield R, Farley MM, Mohle-Boetani J, Harrison LH, Petit S, et al. Epidemiology of invasive group B streptococcal disease in the United States, 1999-2005. JAMA. 2008;299(17):2056-65. doi:10.1001/jama.299.17.2056.

62. Capanna F, Emonet SP, Cherkaoui A, Irion O, Schrenzel J, Martinez de Tejada B. Antibiotic resistance patterns among group B streptococcus isolates: implications for antibiotic prophylaxis for early-onset neonatal sepsis. Swiss Med Wkly. 2013;143:w13778. doi:10.4414/smw.2013.13778.

63. Public Health England. Pyogenic and non-pyogenic streptococcal bacteraemia (EWNI, 2013). United kingdom: PHE2014 Contract No.: 44.

64. Chen K, Puopolo K, Eichenwald E, Onderdonk A, Lieberman E. No increase in rates of early-onset neonatal sepsis by antibiotic-resistant group $B$ streptococcus in the era of intrapartum antibiotic prophylaxis. Am J Obstet Gynecol. 2005;192:1167-71.

65. Kenyon S, Hagberg H, Norman JE. Preterm labour, antibiotics, and cerebral palsy. United Kingdom: RCOG; 2013.

66. Kenyon S, Pike K, Jones DR, Brocklehurst P, Marlow N, Salt A, et al. Childhood outcomes after prescription of antibiotics to pregnant women with preterm rupture of the membranes: 7-year follow-up of the ORACLE I trial. Lancet. 2008;372(9646):1310-8. doi:10.1016/S0140-6736(08)61202-7.

67. Ohlsson A, Shah VS. Intrapartum antibiotics for known maternal group B streptococcal colonization. Cochrane Database Syst Rev. 2014:6:CD007467. doi:10.1002/14651858.CD007467.pub4

68. Taminato M, Fram D, Torloni MR, Belasco AGS, Saconato H, Barbosa DA Screening for group B streptococcus in pregnant women: a systematic review and meta-analysis. Revista Latino-Americana de Enfermagem. $2011 ; 19: 1470-8$

\section{Submit your next manuscript to BioMed Central and we will help you at every step:}

- We accept pre-submission inquiries

- Our selector tool helps you to find the most relevant journal

- We provide round the clock customer support

- Convenient online submission

- Thorough peer review

- Inclusion in PubMed and all major indexing services

- Maximum visibility for your research

Submit your manuscript at www.biomedcentral.com/submit
) Biomed Central 